\title{
Modeling of 2D Photonic Crystal based 1x4 All Optical Splitter
}

\author{
Bani Gandhi* and Anil Kumar Shukla
}

Amity Institute of Telecom Engineering and Management, Amity University, Sector 125, Noida - 20313, UttarPradesh, India; bani.gandhi@gmail.com, akshukla2@amity.edu

\begin{abstract}
Objectives: In this paper a $1 \times 4$ optical beam splitter with a power splitting ratio of 80:20, based on 2D photonic crystal has been modeled for TE-polarized light. Methods: Designing of prototypes was done on the OptiFDTD software. Further, the simulations were performed and post-simulation the results were analyzed, this included the transmission spectra of the design which showed that the power was divided in a ratio of $80: 20$ that is $80 \%$ and $20 \%$ of the transmitted signal. Also, the photonic band gaps were calculated using the Plane Wave Expansion (PWE) band solver. Findings: This prototype comprises of 2D Hexagonal lattice where the elliptical Si-rods are lined up in the air. The size of the wafer used for designing the prototype was taken to be $21 \mu \mathrm{m}^{*} 15 \mu \mathrm{m}$.This particular design is a H-shaped splitter Finite Difference Time Domain (FDTD) and Plane Wave Expansion (PWE) methods are the mostly used mathematical analysis methods that are used for analyzing and calculating the band gap configuration and calculating the Photonic Band Gap of the photonic crystals. The Plane Wave Expansion method calculates the Eigen frequencies and dispersal characteristics of photonic crystals, with the help of Maxwell equations in frequency domain. The output power at output 1 and 3 was $80 \%$ and at the output 2 and 4 was calculated to be $20 \%$ of the transmitted signal. With the tolerance as 0.001 , three band gaps were calculated and the values of their ban gaps were found to be $0.235179,341851 ; 0.459574,0.575928 ; 0.616383,0.628378$.

Novelty/Improvement: This structure is designed in $1 \times 4$ dimensions, further the structure can be designed in various dimensions like $1 \times 8,1 \times 16,1 \times 32$ etc., depending on different applications.
\end{abstract}

Keywords: Optical Splitter, Photonic Crystals, FDTD Simulation, PWE Band Solver

\section{Introduction}

Integrated Technology for optical base modules has developed within optical fiber communications so that it is at the present feasible to fabricate a whole system onto a solo chip. Integration for such devices has become a confluence of numerous photonic or optical disciplines ${ }^{1}$. Electronic control of the optical devices determines the terminology of IO while photons direct the process of IP devices. In addition, IP does not engross any optoelectronic conversion of optical signals and therefore this technology is also termed as 'All-optical'.

Optical splitter which is based on a quartz substrate of an integrated waveguide optical power distribution component, which works on the similar lines of a coaxial cable transmission system ${ }^{2}$. The requirement and desire for using optical power splitters is emerging worldwide as shown in Figure 1, due to the fact that there is an expeditious implementation of fiber-to-the-home, fiberto-the-premises, active optical cables for TV/Video signal transport and optical metropolitan area network (MAN). There is an integral role played by Optical Power Dividers in passive optical network (PON) Technology which facilitates numerous users to share a common optical line terminal.

"Photonic Crystals" are the dielectric compositions which have intermittent spatial variations of the index of

${ }^{*}$ Author for correspondence 
refraction on the dimensions of the wave length of lightwave and the transmission of EM waves is prohibited for the waves within this band gap. During the last several years, more than a few foremost technological applications such that management of trapping of photons and light emanation and transmission, has been accomplished because of the photonic band gap and artificially offered defects $s^{3}$. PCs are defined as structures with periodically varying dielectric constant in special symmetric course with periodicity of the order of the wavelength of the light within the material that the PCs are fabricated from. In line with the periodic dimensions, Photonic Crystals are categorized into one-dimensional (1D), this means the variations in the dielectric are along a single path. In a similar way two-dimensional (2-D) and thirddimensional (3-D) PC's are explained whilst the dielectric properties vary in double and three paths respectively $y^{4}$. The Figure 2 shown in depicts the samples.

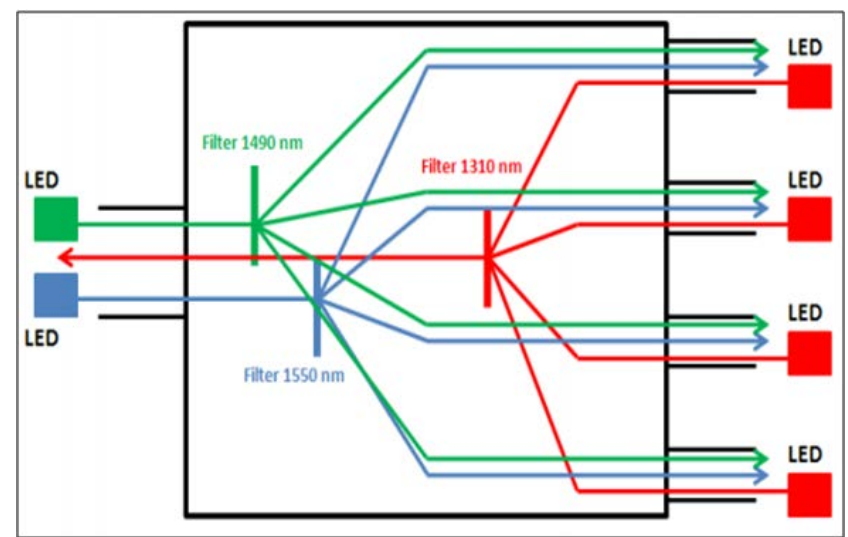

Figure 1. Symbol for a 1X4 power splitter.

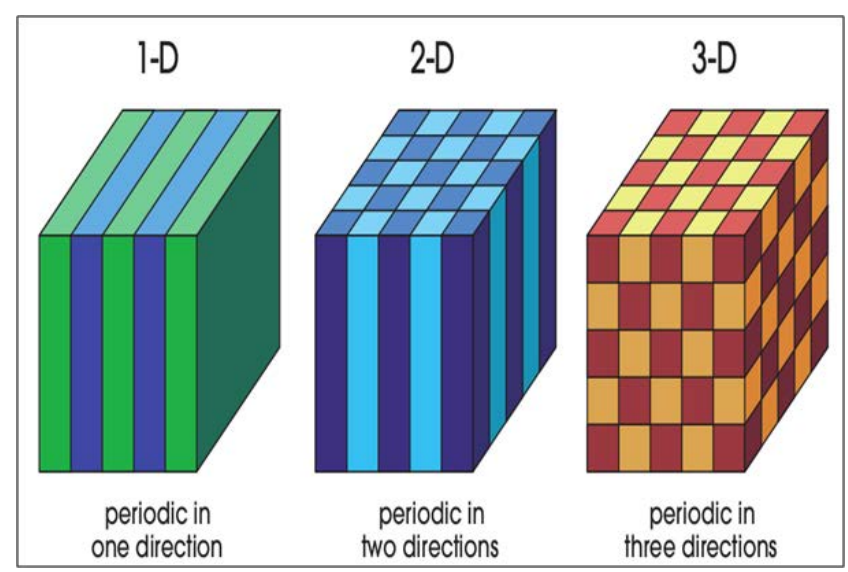

Figure 2. 1D, 2D, 3D Photonic crystals.

\section{Layout Design and Simulation}

The size of the $\mathrm{SiO}_{2}$ lattice is taken to be $21 \mu \mathrm{m}^{\star} 15 \mu \mathrm{m}$ in length and width respectively. Dielectric material was chosen to be air with the refractive index of 1.The input signal is of wavelength of $1.55 \mu \mathrm{m}$ and the mode was selected to be Gaussian Modulated Continuous Wave. An elliptical waveguide and 2D Hexagonal lattice is selecTEd for the model and the value of radius was taken as $0.3 \mu \mathrm{m}$ in Figure 3.

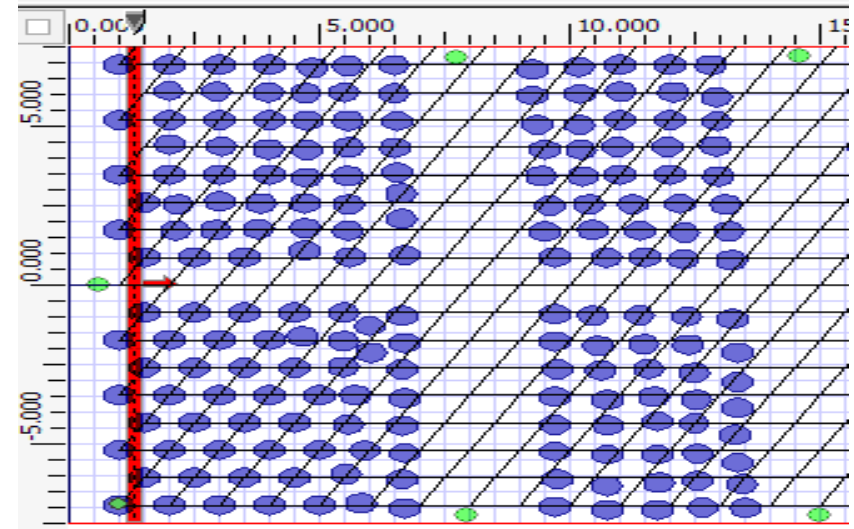

Figure 3. 1x4 H-shaped splitter.

FDTD is a time-domain numerical technique which is used to study the simulation of the design and used for modeling the transmission of EM waves for calculating the Maxwell's equations. FDTD Technique has been found to be very exact and precise for the analysis of the photonic crystal ${ }^{5}$.

The simulation was carried out for TE mode. The boundary condition used for the simulation was Anisotropic Perfectly Matched Layer (APML). Boundary conditions are critical to analyze PC structure since any computational resource can only deal with finite size of matrix and the simulation region has to be terminated by boundaries. The time steps were taken to 1000. FDTD method has two primary advantages; first, this method computational requirements scale is linear, that permits the analysis of superior, asymmetrical configurations in adding up to perfectly periodic structures. Second, this method is simple to implement for complicated scattering structures. The simulation result is shown as Figure 4 .

Physical specifications used to design the structure are mentioned in the Table 1. 


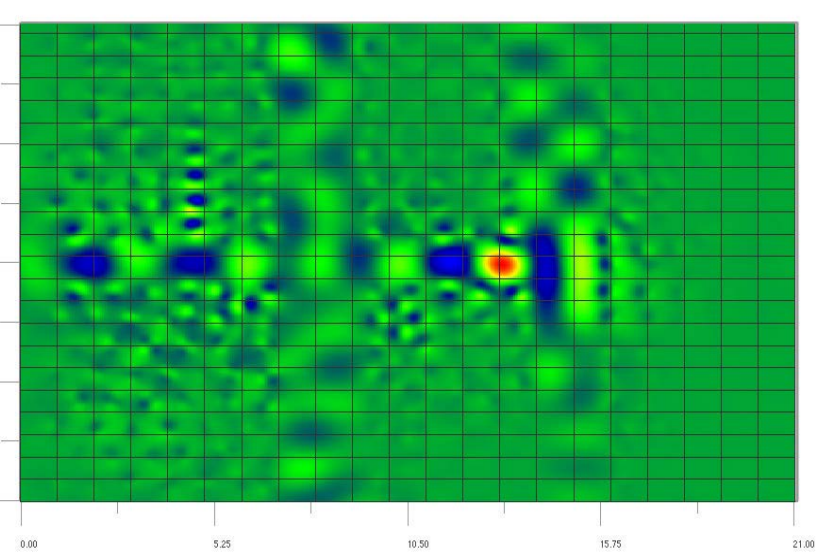

Figure 4. Simulation result of $1 \times 4 \mathrm{H}$-shaped splitter.

\section{Results and Discussions}

\subsection{Power Spectrum}

The power distribution or the power spectrum for the above designed beam splitter is achieved after simulating the design for the TE mode for 2D simulation. One observation point was placed at the input and four were placed to check the response. FDTD technique which uses Discrete Fourier Transform (DFT) for the calculations to obtain the power distribution or the transmission spectrum for the above designed splitter. After the simulation has finished the FDTD Analyzer opens and a graph is generated depicting the input and output response as shown in Figure 5 .

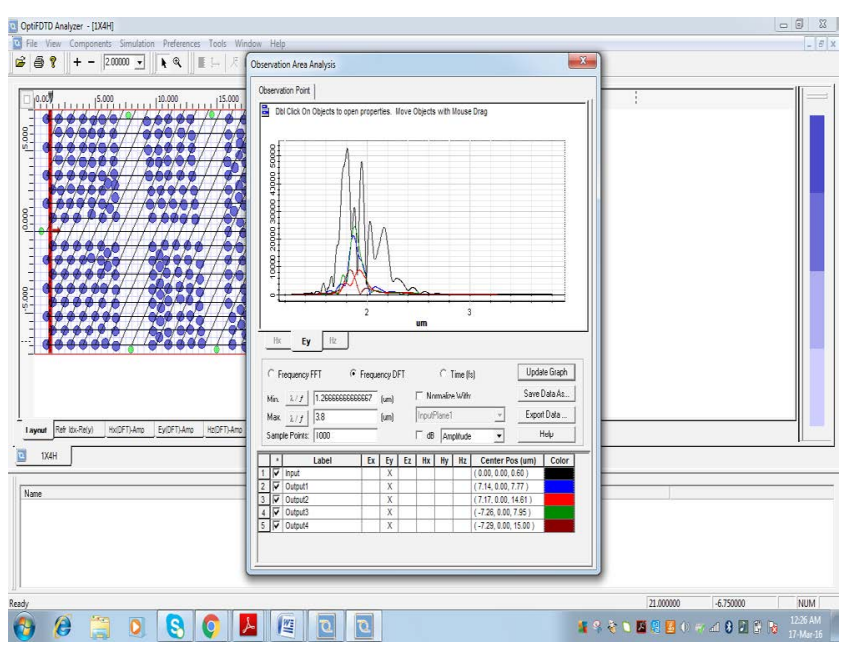

Figure 5. Power distribution or transmission spectrum of all the outputs in $1 \times 4 \mathrm{H}$ shaped splitter.
It can be seen from the graph that the transmitted power at output 1 and 3 was $80 \%$ and at the output 2 and 4 was calculated to be $20 \%$ of the transmitted signal. There is an efficient transmission of the input at all the four ports as that can be seen above.

Table 1. Physical specifiactions of the splitter

\begin{tabular}{|l|l|}
\hline Parameters & Y-Junction Splitter \\
\hline Configuration & Holes with Hexagonal Lattice \\
\hline $\begin{array}{l}\text { Device Size (Length(um) } \\
\mathrm{X}^{\star} \text { Width(um)) }\end{array}$ & $21^{\star} 15$ \\
\hline Band Gaps & 3 \\
\hline Wavelength & $1.55 \mu \mathrm{m}$ \\
\hline Radius of Rods & $0.3 \mu \mathrm{m}$ \\
\hline Refractive index of the rods & 3.4 \\
\hline
\end{tabular}

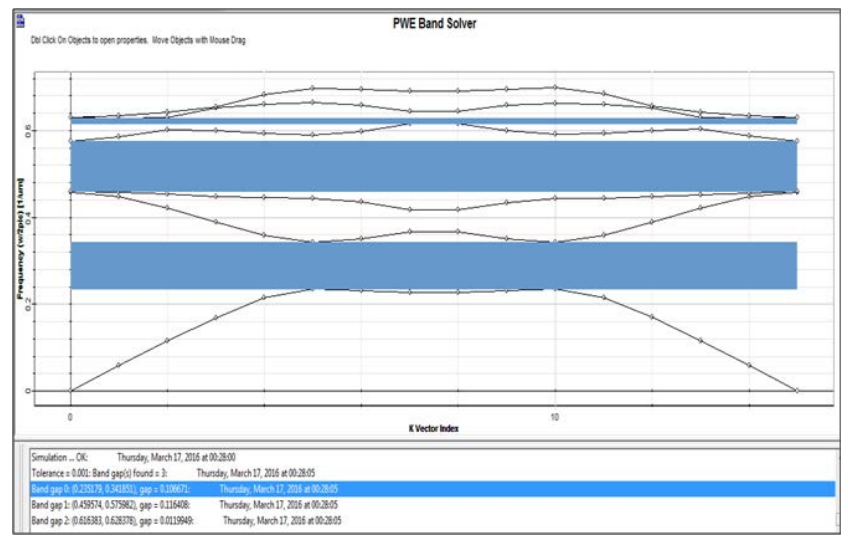

Figure 6. Band gap diagram for $1 \mathrm{x} 4 \mathrm{H}$ shaped beam splitter.

\subsection{Plane Wave Expansion Technique}

Intended for the computation of the photonic band gap and locate the propagating modes of the photonic crystal, the PWE scheme is implemented in most of the cases because of its consistency and proficiency. This method gives the band gap structure to observe various bands and the gap between them (Figure 6). Band gap can be calculated for both transverse electric and transverse magnetic polarizations $s^{z}$. This method is used to validate that how the light will pass thorough this particular configuration. For the band gap analysis the plane wave expansion specifications like the TE polarization mode was taken to be TE then the k-vector path has to be specified and for this it was taken to be square XZ. Below is the graph which 
shows the band gap for the above designed splitter. With the tolerance as 0.001 , three band gaps were calculated and the values of their band gaps were found to be 0.235179 , $341851 ; 0.459574,0.575928 ; 0.616383,0.628378$.

\section{Conclusion}

In this research, evaluation and probing beam splitter based photonic crystal was carried out. The FDTD method was used to calculate the he power distribution at each output port. The Plane Wave Expansion Technique was used to measure the Photonic band gap. It was observed that the output power at output 1 and 3 was $80 \%$ and at the output 2 and 4 was calculated to be $20 \%$ of the transmitted signal. This particular optical splitters can be used in applications that need the STM-1 or SDH optical signal input to be concurrently linked to an active link, while at the same time connecting it to «shadow» equipment such as an STM-1 Groomer or an analyzer, to non-intrusively monitor the «live» STM-1 traffic links. The Optical Splitters also allow the users to non-intrusively test and collect data from «live» STM-1 circuits also this design may be used for power combining and splitting application for quadrature multiplexing, transmitter, receiver and antenna power combining applications. Further, enhanced performance can be obtained by improving the range of design parameters conditions.

\section{Acknowledgement}

The authors sincerely acknowledge the support provided by the family, friends and mentors. We would also like to express our gratitude to the reviewers for their insightful comments which have helped improve this paper.

\section{References}

1. John MS. Optical fiber communications principles and practice. 3rd Edition. Pearson Education Limited: England; 2007. p. 1-1127.

2. Rajat D. Optical power splitting techniques using photonic crystal line defect waveguides. The University of WesTErn Ontario, London, Canada; 2011. p. 1-139.

3. Joannopoulos JD, Johnson SG, Meade RD, Winn JN. Photonic crystals: Molding the flow of light (Princeton University Press, Princeton, NJ); 2011.

4. Hojo H, Uchida N. Control of electromagnetic waves by 2-D plasma photonic crystals. The Joint 30th International Conference on Infrared and Millimeter Waves and 13th International Conference on Terahertz Electronics, IRMMW-THz. 2005; 1:117-18.

5. OptiFDTD Technical Background and Tutorials. Finite Difference Time Domain Photonics Simulation Software; 2011. p. 1-462.

6. Ghafari A, Javid M, Monifi F, Abrishamian MS. A numeric analysis of photonic crystal tunable add-drop filters based on ring resonators.Lasers and Electro-Optics Society. LEOS; 2007. p. 351-2. https://doi.org/10.1109/ LEOS.2007.4382422

7. Kaur H, Kaler RS. Design and analysis of 2D photonic crystal devices. Ph.D Thesis, Thapar University. India; 2015. p. $1-73$. 